\title{
Les besoins en information du personnel des entreprises en Côte d'Ivoire
}

\section{Yahaya Diabi}

\section{(2) OpenEdition}

1 Journals

Édition électronique

URL : http://journals.openedition.org/communicationorganisation/2231

DOI : 10.4000/communicationorganisation.2231

ISSN : $1775-3546$

Éditeur

Presses universitaires de Bordeaux

Édition imprimée

Date de publication : 1 mai 1999

ISSN : 1168-5549

Référence électronique

Yahaya Diabi, «Les besoins en information du personnel des entreprises en Côte d'Ivoire », Communication et organisation [En ligne], 15 | 1999, mis en ligne le 26 mars 2012, consulté le 19 avril 2019. URL : http://journals.openedition.org/communicationorganisation/2231 ; DOI : 10.4000/ communicationorganisation.2231

Ce document a été généré automatiquement le 19 avril 2019

(c) Presses universitaires de Bordeaux 


\title{
Les besoins en information du personnel des entreprises en Côte d'Ivoire
}

\author{
Yahaya Diabi
}

\section{Introduction générale}

\section{Position du problème}

1 La société politique moderne de l'Information des citoyens par les pouvoirs publics est sujette, aujourd'hui, aux instruments de diffusion collective : presse écrite, radiodiffusion et télévision.

2 À l'instar de cette exposition aux médias de masse, les entreprises modernes des pays développés, depuis quelques années, pour l'Information permanente de leurs salariés sur leurs propres activités, leurs résultats, leurs politiques, leurs projets, ont recours à trois sous-systèmes de fonctionnement. Il s'agit des sous-systèmes de direction, de production et d'information pour ce qu'il est convenu d'appeler: Information Générale, Information Opérationnelle et Information administrative du personnel. Ces catégories informatives trouvent leur réalisation en amont par la collecte et le traitement et en aval par une documentation bien élaborée par le personnel d'exécution qui y retrouvera ses besoins, ses engouements, ses attentes par rapport à lui-même d'une part, et d'autre part à la vie de son entreprise où il passe le plus clair de son temps de travail et même de sa vie d'homme.

3 Et cette interaction informative en entreprise s'apparente à la communication d'État par les pouvoirs publics en direction des citoyens tant dans un contexte politique multipartisan qu'unipartisan des États modernes.

4 Venons-en maintenant à la définition et à l'orientation de notre sujet sur : « Les besoins en information du personnel d'entreprise en Côte d'Ivoire ». 


\section{Définition et Orientation du sujet}

5 Selon Albou Paul' ${ }^{1}$, le concept de besoin, c'est la subjectivité d'un désir, c'est aussi la nécessité contrainte à agir pour se satisfaire, c'est aussi la relativité de cette nécessité qui n'est pas partagée par tous.

Aussi le besoin apparaît-il comme l'écart établi entre ce qui devrait être assouvi et qui est ressenti comme un manque momentané. Le besoin est alors, à notre sens, une aspiration subjective qui peut se révéler comme intersubjective c'est-à-dire partagée par tous, devenant aussi une aspiration objective.

C'est pourquoi, dans le cadre de cette communication, nous serons amené à établir des analyses inspirées des besoins de communication sociale d'État dont nous retrouverons les manifestations en entreprise.

Par le concept d'Information nous entendons ici traiter de l'Information Générale données sur l'entreprise, ses objectifs, ses résultats, sa vie, son environnement politique, économique, social et culturel - de l'Information Opérationnelle - faits sur les fonctions des uns et des autres et leur exécution dans les conditions optimales - et enfin l'Information Administrative - données sur les statuts, les profils de carrière, les réunions de toutes sortes, les rémunérations catégorielles, qui ont recours aux écrits professionnels descendants et ascendants.

Par le concept Personnel nous prenons en compte aussi bien le personnel dirigeant que le personnel d'exécution, solidaire dans l'accomplissement des tâches de direction, de production et d'Information d'entreprise.

10 Enfin le concept Entreprise fait ici appel à toute organisation au sens le plus large. Elle concerne les organisations ou institutions publiques, para-publiques et privées où l'acte d'Information est fondé sur une forme de relation sociale. Pourvu que celle-ci soit engendrée par la participation consciente des individus ou des groupes, solidaires pour la réalisation des biens et services au profit des usagers (entreprises publiques) ou de la clientèle (entreprises para-publiques et privées).

11 Pour apporter davantage d'éclairage à notre sujet proprement dit, notre objectif sera :

$121 /$ de décrire et d'analyser le fonctionnement de l'Information d'État à travers les instruments de diffusion collective à l'attention des citoyens des États modernes, administrés par des pouvoirs publics. Il s'agira pour nous de montrer la nature de ces moyens de communication de masse ainsi que les véritables destinataires de ceux-ci. Face à cette interaction informative, l'entreprise nationale ou internationale n'est pas aujourd'hui exempte. Car elle se nourrit et s'enrichit du travail de son personnel qui est sujet à la "sur-information » d'État sur l'État et qui n'entend pas être "sous informé » dans son entreprise, sur son entreprise, et par son entreprise.

2/d'établir une véritable comparaison entre le fonctionnement de l'Information d'État et celle d'entreprise. Car les Sciences de l'Information et de la Communication, dans nos pays du Sud, ont eu à s'intéresser davantage à la communication sociale. Celle d'entreprise y apparaît progressivement à l'instar de la France où c'est au long des années 1980 que les chefs d'entreprise se sont tournés résolument vers la communication d'entreprise alors que les États-Unis et le Japon avaient déjà une longueur d'avance, d'où la compétitivité et l'agressivité reconnues à travers le monde de leurs sociétés. 


\section{L'information d'État comme modèle d'information d'entreprise}

14 Le développement de l'Information à travers les instruments de diffusion collective radiodiffusion, télévision et presse écrite - est une exigence sociale pour le fonctionnement d'un État.

15 En effet, André De Laubadère ${ }^{2}$ distingue trois catégories d'information auxquelles les nations modernes ont recours. Il s'agit de l'information administrative, de l'information nationale et de l'information générale.

\section{Présentation de l'Information Administrative d'État}

Cet auteur présente l'Information Administrative d'État en ces termes: Aucune contestation ne peut évidemment s'élever sur la légitimité de l'intervention de l'État en vue d'informer les citoyens des mesures gouvernementales et administratives qui les concernent. Le Journal Officiel, les Bulletins Officiels des diverses administrations correspondent à cette fonction. À cet égard le principe traditionnel est comme dans beaucoup de pays, celui du caractère confidentiel des documents administratifs, sauf exceptions prévues par des textes ${ }^{3}$.

En Côte d'Ivoire, en Tunisie et comme partout ailleurs dans les états modernes paraissent des publications périodiques qui divulguent les actes gouvernementaux, Ministère par Ministère. La publication de ces informations qui participent aux rapports gouvernantsgouvernes se fait principalement dans une presse écrite dite Journal Officiel ou Bulletin Officiel (d'État) dont la distribution est limitée à l'élite administrative : ministres, préfets ou gouverneurs de région, sous-préfets, directeurs généraux d'administration et de société et autres hauts fonctionnaires.

Les besoins en informations des citoyens sont dans ces conditions, pris en compte par les gouvernants avec la parution régulière de ce type de publication qui reste élitiste certes, mais dont le contenu a trait au politique, c'est-à-dire au fonctionnement de l'État à travers les rapports entre citoyens et décideurs politiques et administratifs. L'élitisme du support imprimé du Journal Officiel ou du Bulletin d'un État réside aussi dans le réseau de la communication de ce type de presse écrite que la masse des citoyens ne se procure pas en surfaces publiques de vente ou dans un centre de documentation ouvert au public.

En revanche, l'Information Nationale, elle, se présente comme un moyen ou un contenu informatif beaucoup plus ouvert aux citoyens que la catégorie d'information susmentionnée.

\section{Les Caractéristiques de l'Information Nationale}

Selon le même auteur précité, l'Information Nationale concerne : «l'Information du public par l'État sur sa propre action, sa politique, ses projets, Information généralement complétée aujourd'hui par une plus large information sur le pays lui-même [...] ».

En Côte d'Ivoire, en Tunisie et partout ailleurs dans le monde des exemples d'instruments de diffusion collective d'Information Nationale existent.

22 En effet, les citoyens de nos états modernes s'exposent à la presse écrite, à la radiodiffusion, à la télévision pour y retrouver leurs attentes en Information 
événementielle, en éducation, et en distraction. L'État, à cet égard, a tout le loisir de recourir à chacun de ces supports pour divulguer l'Information Nationale, telle que présentée par André De Laubadère, aux populations qui ont besoin d'être informées périodiquement sur les politiques mises en œuvre ainsi que sur l'état des projets de changement social qualitatif correspondant à leurs besoins vitaux, identifiés en amont par une recherche sur le terrain.

En Côte d'Ivoire, La lettre de la Primature (organe officiel du Cabinet du Premier Ministre), La lettre de la communication (organe officiel du Ministère de la Communication) et Terre et Progrès du Ministre de l'Agriculture et des Ressources Animales sont autant d'exemples de publications semi-publiques où il est fait état de tel ou tel projet socio-économique, de telle ou telle technique agro-alimentaire plus qualitative que celle qui est déjà en usage.

Cette information nationale, qui s'appuie ici sur le support imprimé à l'attention des cadres et agents de développement, est traitée également au niveau de la radiodiffusion et de la télévision à travers des rubriques spécialisées, susceptibles de prendre en compte les mots d'ordre de mobilisation pour atteindre tel ou tel objectif de développement socioéconomique.

À l'inverse de la presse d'information administrative dont les destinataires sont circonscrits à l'élite de l'appareil politique et administratif, celle d'information nationale a un public-cible plus élargi. En ce sens que ce sont les opérateurs sur le terrain, en contact avec les bénéficiaires des projets mis en œuvre, qui sont les premiers récepteurs de ce type d'information à vocation davantage socio-économique tant en milieux urbain que rural.

En revanche, l'Information Générale, elle, est aussi composite dans son contenu que dans son audience.

\section{L'Information Générale d'État}

Enfin, rappelons toujours André De Laubadère qui définit ainsi la troisième catégorie d'information d'État «l'Information Générale, c'est le problème du rôle de l'État et la légitimité de son intervention dans l'Information Générale (c'est-à-dire l'Information des citoyens sur l'ensemble des événements) qu'il s'agisse de la collecte des informations ou de leur diffusion $»^{5}$.

À la lumière de cette définition, les quotidiens, les hebdomadaires et autres périodiques ivoiriens gouvernementaux ou de partis de l'opposition voire apolitiques, font partie de la catégorie de presse écrite d'Information Générale. Les autres instruments de diffusion collective en Côte d'Ivoire - radiodiffusion et télévision - en font partie. Il s'agit de la première chaîne de la télévision ivoirienne ainsi que de la deuxième. Il en est de même des première et deuxième chaînes de la radiodiffusion nationale.

Les stations de Radiodiffusion Nostalgie (radio davantage distractive) et Espoir (émissions exclusivement catholiques) ne font pas partie de la catégorie de radiodiffusion d'information générale par leur contenu davantage réservé à des rubriques musicales et publicitaires des plus abondantes (il y existe cependant quelques flashes d'information) pour la première, et à des émissions et musiques beaucoup plus religieuses pour la seconde.

30 L'information générale, à laquelle nous faisons allusion ici, participe de l'idée du droit des citoyens de tous bords socio politiques, de toutes catégories sociales, à bénéficier de la 
réception de telle ou telle rubrique d'information - politique, économique, sociale, culturelle, distractive et sportive - à travers la presse écrite, la télévision et la radiodiffusion nationale, régionale, locale (radio et télévision communautaires). Il s'agit là d'instruments de diffusion collective que nombre d'entreprises modernes de taille nationale ou internationale s'emploient de nos jours à introduire en leur sein pour faire comme le pouvoir central d'État.

\section{La similitude entre les catégories d'information d'état et d'entreprises}

31 L'organisation et la gestion de l'information d'entreprise s'inspirent de celles des instruments de diffusion collective d'État dont nous venons de présenter les caractéristiques et les finalités.

En effet, il est courant de voir en Côte d'Ivoire des demandes, des besoins d'information exigés par le personnel des entreprises toutes catégories socio-professionnelles confondues. Car il devient incompréhensible que celles-ci en tant que citoyens d'un État soient « sur-informées » par les organes d'information - presse écrite, radiodiffusion et télévision - et qu'elles soient " sous-informées » par la direction de leur entreprise par quelque moyen de diffusion que ce soit.

33 Face à cette réalité de droit à l'information d'État qui inspire celle d'entreprise, le citoyen devenu personnel - cadre, employé ou ouvrier - attend de ses dirigeants d'être informé sur son entreprise. Il s'agit pour lui d'avoir accès à l'Information Administrative, à l'Information Opérationnelle et à l'Information Générale que son entreprise, à l'instar du pouvoir d'État, doit s'employer à lui fournir régulièrement.

\section{La Corrélation entre le besoin d'information générale d'entreprise et celui d'État}

34 À l'instar des publications d'information générale auxquelles nous venons de faire allusion, nombre d'entreprises modernes ayant un personnel assez étoffé font paraitre des titres quotidiens - SIR Info, de la Société Ivoirienne de Raffinage, bimensuels, Tadji, qui signifie pétrole, de la même société, mensuels, La Source, de la Société d'Eau de Côte d'Ivoire - pour ne citer que ces quelques exemples en Côte d'Ivoire à propos d'entreprises à dimension nationale et internationale.

L'usage du support imprimé, pour l'information générale d'entreprise qui s'apparente dans ses rubriques à celle d'État, se trouve parfois consolidé par la mise en place d'un circuit fermé de Télévision qui diffuse son programme pendant les repas de 12 heures à $14 \mathrm{~h} 30$ à la cantine réservée à tout le personnel de la Société Ivoirienne de Raffinage. À la Société d'Eau de Côte d'Ivoire, les besoins d'information générale du personnel sur toute l'étendue du territoire ivoirien prennent leur appui dans la mise en œuvre d'un système Télé-Radio omnidirectionnel, installé dans chaque direction départementale de cette société. Et ce dispositif technologique communicationnel d'entreprise facilite le décloisonnement des relations inter-services et contribue de cette façon au vécu de l'esprit de corps, de l'esprit maison. En ce sens que tout le personnel de la société se sent rapproché par cette modalité de communication indirecte radiodiffusée par des fréquences spécifiques attribuées par l'Agence des Télécommunications de Côte d'Ivoire. 
Il reste que le besoin d'information opérationnelle d'entreprise n'est pas sans rapport avec l'information nationale dont nous venons de présenter plus haut les tenants et les aboutissants.

\section{Les affinités entre le besoin d'Information Opérationnelle d'entreprise et celui de l'Information Nationale}

37 Il existe en entreprise un besoin permanent de circulation de l'information opérationnelle. Il s'agit ici de la première information que toute entreprise doit communiquer à son personnel à travers son organigramme en prenant en compte la description des tâches respectives des uns et des autres à leur poste précis de travail. Car il s'agit de préciser ici à chaque salarié(e) ce qu'il (elle) doit faire en temps utile et avec le maximum de précisions à son bureau, à son atelier ou à son service.

Ce besoin d'information Opérationnelle descendante est fait d'ordres, d'avis, de directives générales qui expriment somme toute l'impulsion de la direction de l'entreprise.

En échange le premier besoin d'information que tout salarié doit à son entreprise relève de l'information opérationnelle ascendante. Car elle consiste à rendre compte et à proposer, à travers les supports imprimés des rapports d'activité (analytique), des comptes rendus ou procès-verbaux de réunions de travail ou de formation, voire d'information.

40 Face à ce constat, la direction de l'entreprise, saisie de toute cette documentation sur l'Information Opérationnelle, est à même d'apprécier l'efficacité de ses décisions et l'adhésion du personnel aux objectifs choisis. L'expression régulière des besoins de celuici en information opérationnelle par cet échange entre la direction et le personnel à l'exécution, entraîne sa motivation constante à mieux s'intégrer dans le processus humain et technique de production des biens et services.

41 La Société d'Eau de Côte d'Ivoire, la Société Ivoirienne de Raffinage sont les deux principales entreprises en Côte d'Ivoire à privilégier le fonctionnement d'un service de communication. Elles ont compris très tôt la nécessité du vécu d'une véritable communication avec le personnel. Car pour celui-ci, son travail n'est pas le seul déterminant pour son bonheur et le succès de l'entreprise. Ce qui compte le plus pour lui, c'est assouvir ses besoins en information générale comme en information opérationnelle descendante et ascendante sur son entreprise où il passe le plus clair de son temps.

Les caractéristiques de l'information opérationnelle d'entreprise telles que présentées plus haut nous renvoient, en guise de comparaison, à celles de l'information nationale. En ce sens que les deux cas privilégient la mise en œuvre d'actions de projets, de tâches et de politiques initiées déjà à des salariés d'entreprise ou à des citoyens d'une nation.

43 L'objectif est le même dans les deux contextes : répondre positivement aux besoins, aux exigences du personnel ou des citoyens. La réussite du pouvoir central d'État avec son projet de société et son programme de gouvernement et celle d'une entreprise, avec ses stratégies de marketing-mix et ses objectifs organisationnels et personnels sont au prix de la mise en œuvre d'une information opérationnelle ou nationale « bi-univoque ».

Ce mode de circulation de l'information nationale des gouvernants aux gouvernés et viceversa, et de l'information opérationnelle des dirigeants de l'entreprise aux salariés, cadres, employés ou ouvriers et réciproquement, existe en matière d'information administrative tant d'État que d'entreprise. 


\section{Les similitudes entre le besoin d'Information Administrative d'entreprise et celui d'État} la démocratie sociale, fonder son efficience sur la technostructure, mais aussi et surtout sur une organisation participative du personnel avec des cercles de qualité, facteur de dynamisme, de rentabilité constante et d'humanisme inter-personnel et inter-groupe, générateurs de plus-value et de réussites garanties.

\section{Conclusion générale}

51 Les besoins du personnel en information constituent une exigence dans les stratégies et moyens de communication d'entreprise et des organisations.

\section{La communication d'entreprise et des organisations, fille des Sciences de la Communication}

52 Comme nous avons eu à le voir plus haut, la communication d'entreprise consiste à étudier l'ensemble des paramètres et des moyens par lesquels une organisation 
(entreprise, institution, association ou autres structures) systématise sa communication avec son environnement interne (personnel) et externe (partenaires, associés).

\section{西}

Cette communication des organisations s'inspire de celle des instruments de diffusion collective, régis par les pouvoirs publics, d'où notre étude comparative exposée plus haut.

Les entreprises de taille nationale ou internationale et les institutions de toutes natures des pays du Nord sont acquises, il y a maintenant plusieurs décennies, à la civilisation de la diffusion des besoins du personnel à travers les moyens d'information sonores comme le bouche à oreille, l'entretien individuel, les réunions d'information, de travail et de formation, les rencontres de toutes sortes. Il en est de même des supports imprimés avec les notes de services, les circulaires, les rapports écrits, les affiches, les comptes rendus, les écrits d'idées et de suggestions à la direction, les lettres personnalisées des dirigeants d'entreprise à chaque membre du personnel.

5 Les moyens d'information électronique font aussi partie de cette même panoplie avec l'utilisation du téléphone, de la télématique, de la radio, de la télévision, des hautparleurs à des lieux publics d'entreprise, les projections de films, l'internet et l'intranet. Oui, ces deux concepts sont aujourd'hui largement connus. Car ils participent de l'avènement réel du village planétaire mac-luhanien tant en communication d'entreprise que d'État.

56 L'information administrative d'État avec internet est susceptible d'être transmise en temps réel à travers les sièges de tous les pouvoirs centraux de la planète pourvu qu'il y ait la volonté de coopérer à cet effet à l'heure du laisser-aller et du laisser-faire au sein des unions économiques et douanières des différents groupements d'état dans chaque continent. Cette information administrative, miroir des actes et des mesures gouvernementaux, n'est pas sans rapport avec les notes administratives des dirigeants d'entreprise qui sont, dans le cadre d'intranet, diffusés sur les micro-ordinateurs des différents responsables hiérarchiques pour le fonctionnement des services, des bureaux ou des ateliers.

Il s'agit là de faire en sorte que les besoins en informations administratives, opérationnelles ou générales circulent de haut en bas de l'organigramme et vice-versa pour la permanence de la communication humaine. Voilà le premier facteur de motivation du personnel qui concourt davantage à atteindre les objectifs choisis dans le cadre d'une structure participative d'entreprise qui s'appuie sur la technicité de ses spécialistes de tous les palliers socio-professionnels.

Et comme nous avons eu à le souligner plus haut, la communication d'entreprise a le même objet que les Sciences de la Communication au sens le plus large. Car l'expression des besoins de personnel dans une organisation ou dans une entreprise trouve sa similitude avec celle de l'étude des instruments de diffusion collective d'un pouvoir central ou d'une organisation politique. En ce qu'ils ont leurs adhérents, leurs sympathisants à l'instar du personnel d'une entreprise. Et les deux catégories de destinataires s'exposent aux moyens d'Information sonores, imprimés et audiovisuels tels que présentés plus haut. 


\section{La pérennisation et la spécificité de la fonction communication d'entreprise et d'organisation}

\section{d'entreprise plutôt externe, "House organ » (au destinataire davantage externe) et qui} anime une télévision en circuit fermé, comme à la Société Ivoirienne de Raffinage, apparaît comme un progrès notoire dans la reconnaissance de la fonction communication d'entreprise par rapport aux nombreuses entreprises et à des services publics qui sont encore sceptiques à sa création. Seuls et commencer par Les étudiants du Brevet de Technicien Supérieur en communication et actions publicitaires à travers leur association sont les mieux indiqués pour promouvoir cette fonction communication et organisations. L'espoir peut alors permettre de voir à terme les mairies (Cocody, Le Plateau, Port-Bouët, de Bouaké, de Yamoussoukro et d'autres importantes villes de l'intérieur), toutes les préfectures et tous les grands services publics se doter d'une cellule de communication dans un premier temps.

Mais la finalité ultime, c'est d'arriver à mettre sur un pied d'égalité dans l'organigramme la Direction Technique, la Direction du Personnel, la Direction des Etudes avec la Direction de la Communication. Car l'efficacité de cette direction, son utilité sociale, dépendent de la mise en place d'un budget autonome à l'instar des autres directions opérationnelles.

C'est à cette condition, à notre sens, que la connaissance ascendante et descendante des besoins du personnel à travers les supports d'information administrative, opérationnelle et générale trouvera toutes ses forces d'action et de rétroaction positives pour la 
régénération constante de l'entreprise ou de toute organisation sociale. Il s'agit là d'un défi, d'une nouvelle exigence managériale pour les entreprises africaines en général et ivoiriennes en particulier, intégrées déjà dans la mondialisation, faite de compétitivité. Pour s'y maintenir en alter ego, les entreprises des pays du Sud se doivent alors de recourir aux mêmes armes que celles des nations industrialisées où le sous-système d'Information, au sein de leurs sociétés, est tout aussi à l'honneur que les sous-systèmes de direction et de production. Car pour ne citer que l'exemple de la France : selon différentes études $85 \%$ des services de communication sont aujourd'hui rattachés aux directions générales contre $15 \%$ il y a vingt ans.

Et pour atteindre cette finalité dans les pays du Sud, les instituts et écoles de formation en Sciences de la communication devraient refonder leurs programmes de formation pour prendre en compte des modules tels que le marketing-mix, la communication d'entreprise, la communication commerciale, les techniques de communication et de négociation, l'analyse transactionnelle et la programmation neurolinguistique, le droit de (à) la communication, la sociologie des organisations, la sociologie des relations internationales, les outils de l'audit en communication ainsi que la communication de presse technique et professionnelle.

Ce sont là autant de thèmes ou de matières qui visent à former un homme nouveau, un nouveau communicateur, capable de relever les défis de toute entreprise, de toute organisation impliquée dans la société globale de l'information. Faire fi de cette réalité c'est encourir un autre obscurantisme. Les citoyens des pays du Sud ainsi que les personnels de leurs entreprises et de leurs organisations risquent alors d'être des analphabètes des autoroutes de l'information ou pire des non-voyants et des malentendants du III ${ }^{e}$ millénaire, si proche de nous, et si acquis cependant à notre changement social qualitatif par toutes les technologies de communication qu'il offre à l'humanité.

67 Nul n'échappe plus à l'empire des « Nouveaux médias », ces NTIC qui finissent par ne plus être si nouveaux, tout en se perfectionnant chaque jour, et qui étendent leurs ramifications à la planète entière; l'Afrique n'est pas épargnée par le mouvement. Pourquoi le serait-elle, d'ailleurs, à suivre la chronique des bienfaits annoncés? De fait, quelques acteurs particulièrement concernés ${ }^{1}$ développent des discours alléchants sur ce qu'il faut en attendre: le développement des NTIC va bouleverser les situations existantes, et permettre d'accéder à des trésors culturels incommensurables, d'introduire plus de démocratie dans les sociétés, d'intégrer les économies africaines au marché mondial, de sauvegarder les cultures, d'instaurer le dialogue entre groupes sociaux, d'amplifier la recherche, etc. Ce continent dénué entre tous pourrait trouver enfin la solution à tous les maux qui l'accablent, depuis maintenant des décennies, si ce n'est plus.

De fait, globalement, l'Afrique demeure le continent de toutes les difficultés. Elle rassemble les pays les plus pauvres du globe, ceux qui disposent des revenus les plus limités, (revenus monétaires, eau disponible, énergie consommée, ressources alimentaires). C'est toujours le lieu de la plus forte croissance démographique, de la plus faible industrialisation, elle n'assure pas son autosuffisance alimentaire, ses sols se dégradent de manière accélérée, ses ressources naturelles sont pillées. Elle ne représente plus que quelques \% des échanges internationaux, croule sous la dette, et un certain nombre de maux persistants, parmi lesquels la dictature, les conflits dénommés «de basse intensité », mais aussi des inégalités sociales criantes ne représentent que la partie émergée d'un iceberg difficile à cerner ${ }^{2}$. Un certain nombre d'améliorations se produisent 
dans certains secteurs, des réalisations positives viennent en quelque sorte « miter » ce tableau ${ }^{3}$, mais pour l'instant on ne voit pas clairement se dégager un changement global qui inciterait à plus d'optimisme.

C'est dans un tel contexte qu'un certain nombre d'acteurs (géants des télécommunications, organisations bilatérales, multilatérales, etc.) interviennent, depuis déjà quelques années, pour stimuler le démarrage et le développement des techniques de communication les plus avancées, notamment l'accès à Internet, la radiotéléphonie et les communications satellitaires (téléphonie, transmission de données, télévision).

Il est frappant de constater à quel point leur discours est similaire à celui qui a été développé autour de la télévision, puis de l'informatique, ou dans le domaine plus modeste, mais tout aussi symbolique, des systèmes documentaires d'IST. Il s'agit à chaque fois d'introduire et de déployer des techniques investies de la capacité de «faire réaliser un bond dans le XXI siècle", de mettre enfin un terme aux divers dysfonctionnements existants ${ }^{4}$, de remédier aux carences de tous ordres constatables dans la vie de tous les jours.

\section{BIBLIOGRAPHIE}

ALbou Paul, « Sur le concept de besoin », in Cahiers Internationaux de Sociologie, Paris, Presses Universitaires de France, 1975, vol LIX

BARTOLI Annie, Communication et Organisation pour une politique générale cohérente, Paris, Édition d'Organisation, 1991.

BIZOUARD Collette, Vivre la Communication, Paris, Editions Chroniques Sociales de France, 1980. DE LAUBADERE André, Traité de Droit Administratif $3^{\mathrm{e}}$ édition entièrement refondue, Les Grands Services Publics Administratif, Paris, Librairie Générale de Droit et de Jurisprudence, 1978.

DELVILLE André, L'information dans l'Entreprise, Paris, Édition Dunod, 1969.

DE NARBonne Aimery, Communication d'Entreprise, Conception et Pratique, Paris, Édition Eyrolles, 1979. DUFouR Arnaud, Internet, Paris, Presses Universitaires de France, Q.S.J. n 3073,1996.

DUPUY Emmanuel, DEVERS Thomas, et RAYNAUD Isabelle, La Communication interne vers l'Entreprise transparente, Paris, Les Editions d'Organisation, 1988.

GERSTLE Jacques, La Communication Politique, Paris, Presses universitaires de France, Q.S.J. $n^{\circ}$ 2652, 1992. GONDRAND François, L'information dans l'Entreprise, pourquoi ? Comment ? Paris, Les Editions d'Organisation, 1976.

HENRIET Bruno, BONEU François, Audit de la Communication interne, Paris, Les Editions d'Organisations, 1990. WESTPHALEN Marie-Hélène : Le Communicator, guide opérationnel pour la Communication d'Entreprise, Paris, Édition Dunod, 1990. 


\section{NOTES}

1. Albou Paul, «Sur le concept de besoin » pp. 229-236, in Cahiers Internationaux de Sociologie, Paris, PUF, 1975, Vol UX

2. André De Laubadère: Traité de Droit Administratif ( $3^{\mathrm{e}}$ Édition entièrement refondue), Les Grands Services Publics Administratifs, Paris, Libraine Générale de Droit et de Jurisprudence, $1978,563 \mathrm{p}$.

3. Ibid. p. 394

4. Ibid, p. 396. Ibid pp. 395-396

5. Ibid, pp. 395-396.

\section{RÉSUMÉS}

La communication d'entreprise est, aujourd'hui, un phénomène assez courant dans les pays du Nord. Les pays du Sud, depuis les années soixante-dix, sont influencés par cette réalité sociotechnique de l'information et de la communication qui est aussi devenue une donnée importante dans le management des entreprises et des administrations.

Dans ce domaine, la Côte d'Ivoire, pays toujours ouvert au développement et à la modernisation de ses structures publiques, parapubliques et privées, depuis son accession à l'indépendance en 1960, fait figure de proue en Afrique Subsaharienne.

Cette étude porte sur l'avènement de la communication dans les grandes entreprises ivoiriennes pour une meilleure intégration du personnel.

Communication in organizations is, today, a general phenomenon in Northern countries. Since the seventies Southern countries have been influenced by this social and technical reality of information and which have become an important factor in enterprises and administrations management.

In this field, the Ivory Coast (a country which has always been open to development and modemization of its public, Para public and private structures since its independence in 1960) is one of the leading countries of subsahara Africa in accepting this science.

This research focuses on the advent of communication inside big Ivory firms to promote a better integration.

\section{INDEX}

Mots-clés : citoyens, élite administrative, esprit maison, opérateurs, personnel d'entreprise 


\section{AUTEUR}

\section{YAHAYA DIABI}

L'auteur est Doyen de l'UFR Information, Communication et Arts de l'Université de Cocody après avoir obtenu son doctorat en Sciences de l'Information et de la Communication à l'Université de Bordeaux en 1985. Il a participé à des recherches sur les médias. Il enseigne la communication des organisations et encadre des mémoires de maîtrise dans ce domaine. 